\title{
Ipsilateral Hip Dislocation with Knee Fracture Dislocation: A Rare Combination
}

\author{
${ }^{1}$ Assistant Professor, Department of Orthopaedics, Kalpana Chawla Govt Medical College, India \\ ${ }^{2}$ Senior Resident, Department of Orthopaedics, ESI - PGIMSR, India \\ ${ }^{3}$ Senior Resident, Department of Orthopaedics, Kalpana Chawla Govt Medical College, India \\ ${ }^{4}$ Senior Resident, Department of Anesthesia, Kalpana Chawla Govt Medical College, India \\ ${ }^{5}$ Assistant Professor, Department of Orthopaedics, Punjab institute of Medical Sciences, India \\ ${ }^{7}$ Senior Resident, Department of Anesthesia, AIIMS Rishikesh, India \\ ${ }^{8}$ Senior Resident, Department of Medicine, VMMC \& SJH, India
}

Mohit Jindal ${ }^{1 *}$, Agarwal $S^{2}$, Arya $A^{3}$, Garg Keerty ${ }^{4}$, Gandhi $V^{5}$, Gupta $\mathrm{SR}^{3}$, Arya $\mathrm{S}^{6}$ and Garg $\mathrm{A}^{7}$

Submission: August 08, 2019; Published: October 10, 2019

*Corresponding author: Mohit Jindal, Department of Orthopaedics, Kalpana Chawla Govt Medical College, India

Abstract

Simultaneous occurrence of ipsilateral hip dislocation with knee fracture dislocation is a rare combination of injuries caused by high energy trauma with motor vehicle accidents and sporting injuries accounting for maximum number of cases. There is also paucity of literature on these types of injuries so there are high chances of overlooking one injury in the presence of other. The clinician should perform a thorough examination to avoid this error. The patient requires prompt attention, resuscitation and urgent reduction of joint dislocation. The prognosis is excellent if dealt with meticulously, otherwise it may become an ordeal for both the patient and the treating doctor.

\section{Introduction}

Hip Dislocation occurs due to high energy motor vehicle accidents [1] and have been classified into anterior or posterior dislocations with posterior being more common (>90\%). Posterior dislocation is associated with sciatic nerve palsy in approximately $10-15 \%$ of patients and is also associated with osteonecrosis of the femoral head in $1-22 \%$ of cases. The attitude of the limb is flexion, adduction and internal rotation in posterior dislocation and shortened and externally rotated in anterior dislocation. Knee fracture dislocation also results from high energy trauma and may be associated with open wounds, extensive soft tissue injury, compartment syndrome and neurovascular injury. They have been classified based on either the direction of tibial displacement relative to the femur (Kennedy position classification system) or the ligaments disrupted in the process (Schenck anatomic classification system). Here we present a rare case report of patient with ipsilateral hip dislocation with knee fracture dislocation.

\section{Case Report}

A 20 year old male patient came to KCGMC Emergency with history of road traffic accident. The patient had unstable vitals and thready pulse with open wound over the right knee (compound grade 2). The right lower limb was shortened in flexion, adduction and in internal rotation, distal pulsations were non palpable. After initial resuscitation to stabilize vitals, skiagram of the right hip and right knee was done and at the same time got the requisite investigations and arranged blood for transfusion. Skiagram showed posterior hip dislocation (Thompson and Epstein type I) with knee fracture dislocation (Schenck type V) of the right side. The patient was immediately posted for emergency surgery for at least reduction of hip and knee and stabilizes the same with what we have. We were in a dilemma over the treatment protocol as such cases are indeed very rare. We could not reduce the hip joint as traction would not be effective and would have caused more damage to the vascularity as there was knee fracture dislocation also. So, we first reduced the knee joint and fixed the same with 2 Steinmann pins and then reduced the hip using the Allis technique.

Fortunately, we were able to reduce both the hip joint and the knee in the same sitting, but distal pulsations got a little feeble and we abandoned any further procedure and subjected the patient to Color Doppler of the lower limb. After resuscitation, 


\section{Orthopedics and Rheumatology Open Access Journal (OROAJ)}

CT of the hip and knee was done. CT Hip came out to be normal with congruent hip joint with no evidence of any other bony injury. CT of the knee showed proximal tibia fracture involving the lateral condyle (Schatzker type I), which was fixed with two $6.5 \mathrm{~mm}$ cannulated cancellous screws. MRI of the knee was not done as clinically patient does not seem to have any ligamentous instability. Patient was kept in abduction bar and above knee slab for 6 weeks with static quadriceps exercise. He was then mobilized initially with knee range of motion exercises and partial weight bearing. Full weight bearing was allowed after 12 weeks (Figures 1-3).

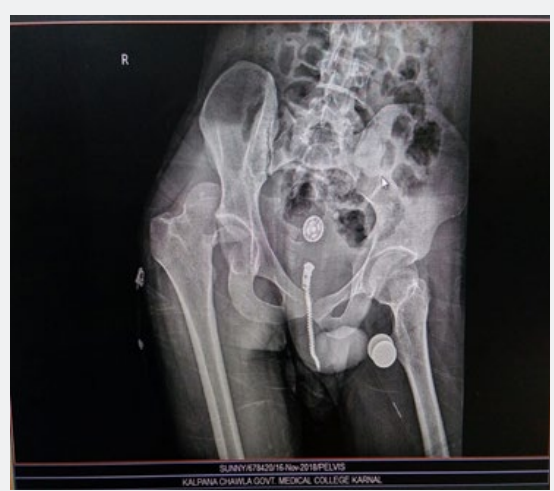

Figure 1: Pre-op X-Ray of Pelvis with bilateral hip joints.

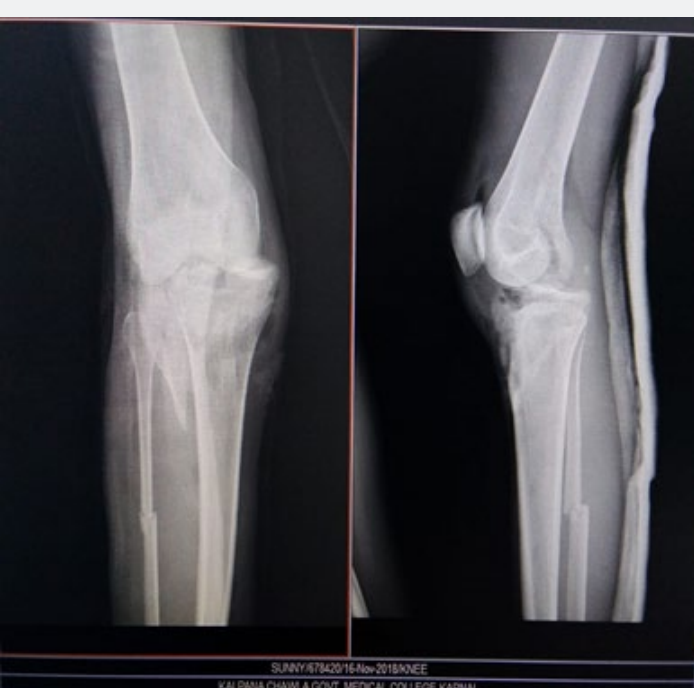

Figure 2: Pre-op X-Ray of Right Knee.

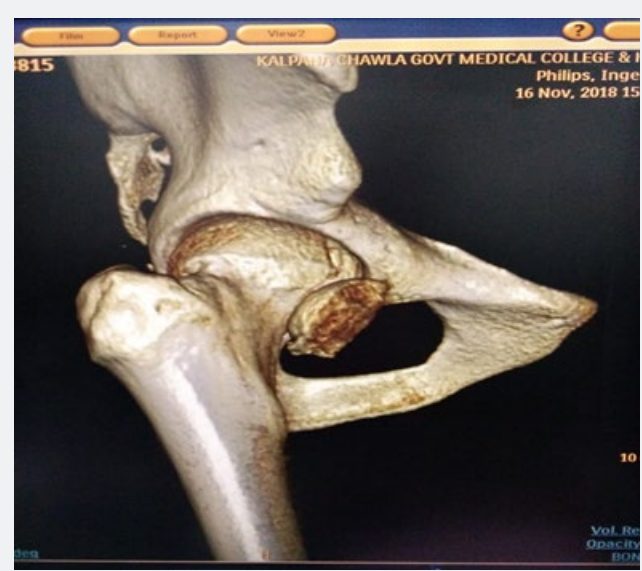

Figure 3: CT Scan Right hip joint after reduction of hip joint. 


\section{Discussion}

Hip and knee dislocations are high energy and high impact injuries that can be debilitating. These injuries are most often seen in motor vehicle collisions and in sports injuries. When hip and knee dislocation present simultaneously, it should be approached as dual emergency as other associated systemic injuries may be present. Posterior hip dislocations are more common than anterior hip dislocations, contributing to around $90 \%$ of hip dislocations and they are often a result of axial force transmitted through a flexed knee, which is commonly seen in dashboard injuries [2]. Anatomy of the proximal femur, position of the hip and direction of the force vector determine whether a pure isolated ligamentous dislocation occurs or an associated fracture with the dislocation.

Posterior hip dislocation has been classified into 5 types by Thompson and Epstein:

i. Posterior dislocation of the hip with or without a minor fracture

ii. Posterior dislocation of the hip with a large single fracture of posterior acetabulum.

iii. Posterior dislocation of the hip with a comminuted fracture of the rim of acetabulum with or without a major fragment.

iv. Posterior dislocation of the hip with fracture acetabular rim and floor.

v. Posterior dislocation of the hip with associated fracture femoral head.

Neurological involvement in hip dislocations occurs in approximately $10 \%$ and partial recovery can be expected in
$60-70 \%$ cases [3]. The peroneal branch of the sciatic nerve is most commonly involved. The ideal time to reduce dislocated femoral head is within 6 hours and no later than 12 hours after dislocation. If the concentric reduction is not achieved with 1 or 2 attempts; open reduction internal fixation (ORIF) with removal of loose intra-articular fracture debris should be considered without a delay [4]. Osteonecrosis of the head of the femur is a serious and unpredictable complication which can occur after posterior hip dislocations due to disturbed blood supply of the femoral head. It is also thought that osteonecrosis is more likely to occur with delayed time to surgical fixation.

Knee dislocations are defined as clinical or radiological loss of tibiofemoral congruity representing less than $0.2 \%$ of orthopaedic injuries. Nearly $50 \%$ of knee dislocations occur due to road traffic accidents with sports injuries accounting for $33 \%$ of dislocations [5]. Knee dislocations can be classified according to Kennedy position classification system (based on the direction of tibial displacement relative to the femur) or Schenck anatomic classification system [5] (based on the ligaments disrupted in the process). The Kennedy system classifies knee dislocation into anterior, posterior, lateral, medial, and rotator. Rotatory dislocations were later subclassified into anteromedial, anterolateral, posteromedial and posterolateral. Schenck classification was developed because Kennedy position classification system does not consider the ligamentous injuries and secondly, up to $50 \%$ of knee dislocations are spontaneously reduced before medical evaluation, hence unclassifiable by the Kennedy classification. Schenk classified knee dislocations in terms of ligaments involved, and this is best done during the time of presentation, or during examination under anesthesia (EUA). This anatomic classification system describes the ligaments torn and is useful in deciding treatment (Table 1).

Table 1: Schenck Anatomic Classification System for Knee Dislocations.

\begin{tabular}{|c|c|}
\hline Type & Description \\
\hline KD I & Knee Dislocation with either cruciate intact \\
\hline KD II & Bicruciate injury with collaterals intact \\
\hline \multirow{2}{*}{ KD III } & Bicruciate injury with one collateral ligament injury \\
\cline { 2 - 2 } & KD IIIM - Bicruciate injury with medial collateral ligament injury. \\
\cline { 2 - 2 } & KD IIIL - Bicruciate injury with lateral collateral ligament injury \\
\hline KD IV & Bicruciate injury with both collateral ligament injury \\
\hline KD V & Periarticular fracture dislocation \\
\hline
\end{tabular}

Associated injuries: C: Arterial injury; N: Neural injury

Knee dislocations have an associated vascular injury in 18\% of cases and neural injury in $14-25 \%$ of cases with common peroneal nerve being most common [5]. Fractures of either the distal femur or proximal tibia are reported to occur in between $4.5 \%$ and $34 \%$ of cases of knee dislocation [6]. The fracture should be treated first, and ACL and PCL injuries may be treated after fracture healing. If continued radiographic and clinical signs of instability are observed, a recommendation for staged reconstruction of ligamentous injury should be considered after bony union has been achieved [7].

Isolated hip or knee dislocation are common, and one can come across them many a time in his orthopaedic career, but simultaneous ipsilateral hip and knee dislocation is a very rare combination. Very few cases have been reported in the 


\section{Orthopedics and Rheumatology Open Access Journal (OROAJ)}

literature. A similar case of simultaneous ipsilateral dislocations of the hip, the knee, and the ankle has been reported by Waterman and Banerjee in 2011 [8]. For recognition of such a pattern, an orthopaedician should undertake meticulous clinical examination as one of the injuries is liable to be missed in the presence of the other. Treatment of these kind injuries pose a significant challenge and may be very grueling as attitude of the injured limb can be misleading. The outcome may also vary depending on other associated injuries and time elapsed before the reduction of the joints; these injuries can potentially have severe complications resulting in impaired function and decreased quality of life (Figures 4-6).

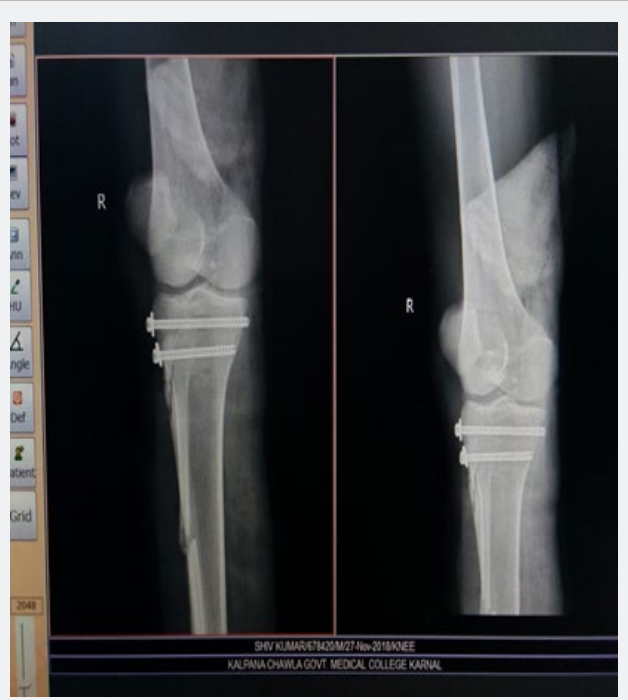

Figure 4: Post-op X-Ray of right knee joint after internal fixation using Cannulated Cancellous Screws.
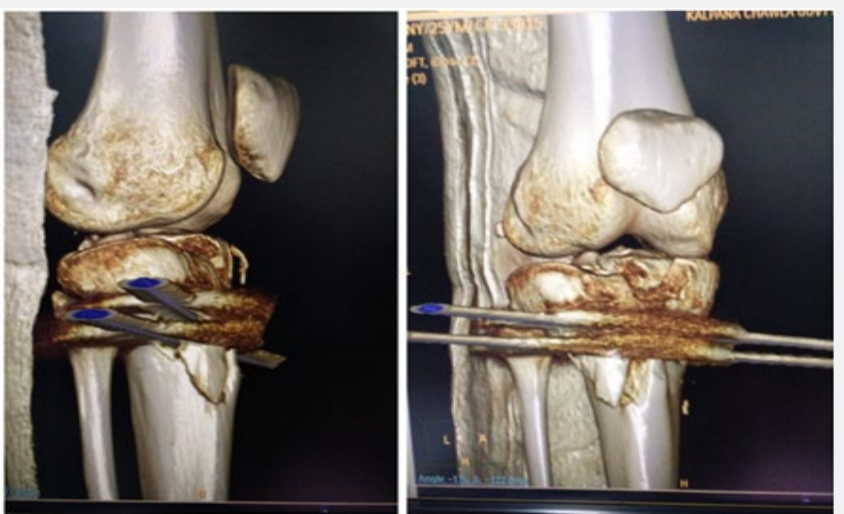

Figure 5: CT Scan Right Knee after reduction and skeletal traction application.

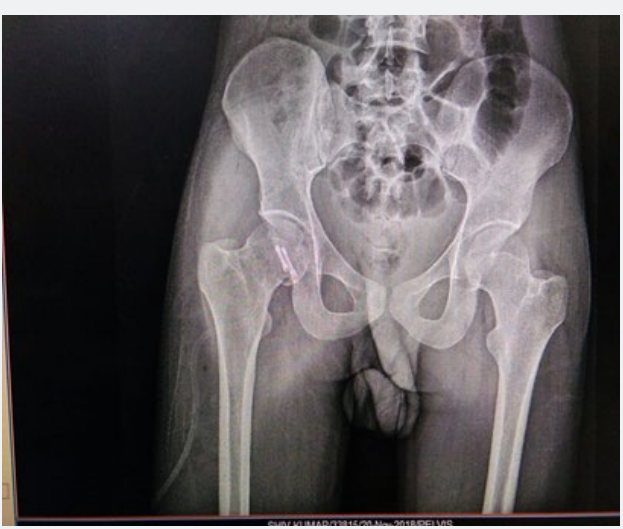

Figure 6: Post-op X-Ray of pelvis with bilateral hips. 


\section{Conclusion}

Dislocation of any joint is treated as an orthopaedic emergency. Hip Dislocation can be associated with concomitant injuries to ipsilateral thigh and knee. These constellations of injuries can likely lead to poor clinical outcomes. The importance of continuous assessment of distal pulses should not be underestimated and vascular injuries should be addressed promptly. The outcome of such patients varies with associated injuries and time took for relocation and risk of avascular necrosis. Treating orthopaedician should have a high index of suspicion for the same to avoid missing these injuries and have a favorable outcome.

\section{References}

1. Misir A (2017) Unusual combination of femoral head dislocation associated acetabular fracture with multipart femoral fractures: Case report. Int J Surg Case Rep 34: 32-35.

2. N LKS, Kumar D, Aggarwal S (2018) Simultaneous traumatic dislocation of the hip knee and ankle joints in an ipsilateral limb, does it happen? A case report. ISJ 5(7): 2660-2663.
3. Perumal R, Sundararajan SR, Vasudeva J, Rajasekaran S (2016) Ipsilateral Hip and Knee Dislocation with Open Tibial Fracture: A Case Report of a Limb Threatening Injury. J Orthop Case Rep 6(5): 47-50.

4. Chotai PN, Ebraheim NA, Hart R, Wassef A (2019) Concomitant Posterior Hip Dislocation, Ipsilateral Intertrochanteric- and Proximal Tibial- Fractures with Popliteal Artery Injury: A Challenging Trauma Mélange. Clin Pract 5(4): 776.

5. Pardiwala DN, Rao NN, Anand K, Raut A (2017) Knee Dislocations in Sports Injuries. Indian J Orthop 51(5): 552-562.

6. Amar MF, Chbani B, Ammoumri O, Marzouki A and Ii H (2010) Open posterior dislocation of the knee with rupture of the patellar tendon and a tibial plateau fracture. Orthopaedic Rev (Pavia) 2(1): e7.

7. Sabesan VJ, Danielsky PJ, Childs A, Valikodath T (2015) Multiligament knee injuries with associated tibial plateau fractures: A report of two cases. World J Orthop 6(3): 363-368.

8. Arenas-miquelez A, Bern L, Familiari F, Arbeloa-gutierrez L, Azzarelli AD (2017) Simultaneous Ipsilateral Dislocation of the Hip and the Knee: A Case Report. JBJS Case Connect 7(4): e85.

\section{Your next submission with Juniper Publishers will reach you the below assets}

- Quality Editorial service

- Swift Peer Review

- Reprints availability

- E-prints Service

- Manuscript Podcast for convenient understanding

- Global attainment for your research

- Manuscript accessibility in different formats

( Pdf, E-pub, Full Text, Audio)

- Unceasing customer service

Track the below URL for one-step submission https://juniperpublishers.com/online-submission.php 